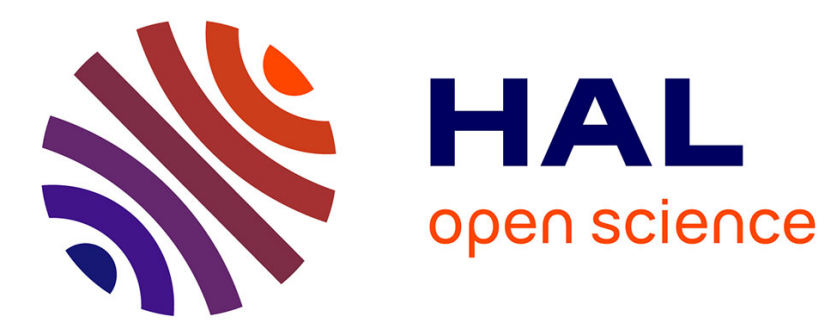

\title{
Incorporation of a Vector Preisach-Mayergoyz Hysteresis Model in 3-D Finite Element Analysis
}

Maxime Tousignant, Frederic Sirois, Gérard Meunier, Christophe Guerin

\section{To cite this version:}

Maxime Tousignant, Frederic Sirois, Gérard Meunier, Christophe Guerin. Incorporation of a Vector Preisach-Mayergoyz Hysteresis Model in 3-D Finite Element Analysis. IEEE Transactions on Magnetics, 2019, 55 (6), pp.7300504. 10.1109/TMAG.2019.2900690 . hal-02350904

\section{HAL Id: hal-02350904 https://hal.science/hal-02350904}

Submitted on 30 Mar 2021

HAL is a multi-disciplinary open access archive for the deposit and dissemination of scientific research documents, whether they are published or not. The documents may come from teaching and research institutions in France or abroad, or from public or private research centers.
L'archive ouverte pluridisciplinaire HAL, est destinée au dépôt et à la diffusion de documents scientifiques de niveau recherche, publiés ou non, émanant des établissements d'enseignement et de recherche français ou étrangers, des laboratoires publics ou privés. 


\title{
Incorporation of a Vector Preisach-Mayergoyz Hysteresis Model in 3-D Finite Element Analysis
}

\author{
Maxime Tousignant ${ }^{\circledR 1,2,3}$, Frédéric Sirois ${ }^{\circledR 1}$, Gérard Meunier ${ }^{\circledR 2}$, and Christophe Guérin ${ }^{3}$ \\ ${ }^{1}$ Polytechnique de Montréal, Montreal, QC H3T 1J4, Canada \\ ${ }^{2}$ Univ. Grenoble Alpes, CNRS, Grenoble INP, G2Elab, 38000 Grenoble, France \\ ${ }^{3}$ Altair Engineering France, 38240 Meylan, France
}

\begin{abstract}
A new formulation of the Preisach-Mayergoyz model was developed to radically improve its numerical performance. This new computationally efficient vector hysteresis model was incorporated in a 3-D finite element software. Many improvements to the transient nonlinear solver were made in order to obtain a good convergence of the TEAM problem 32 while using only 125 time steps per cycle. The results of this simulation show good agreement with the experimental data.
\end{abstract}

Index Terms-Convergence, finite element (FE), magnetic hysteresis, Mayergoyz, Preisach.

\section{INTRODUCTION}

A GREAT deal of effort has already been made to include vector hysteresis models in 3-D finite element (FE) analysis, yet some challenges remain. For example, in a recent work, Hoffmann et al. [1] have reported good results for the simulation of the TEAM workshop problem 32 with a 3 -D vector Jiles-Atherton model. However, in order to obtain these results, they reportedly used as many as 500-time steps per cycle to overcome convergence issues.

This paper focuses on achieving a good convergence with a reasonable time-step size in order to solve the TEAM problem 32 within an acceptable time frame. Instead of using the Jiles-Atherton model, we implemented a new version of the Preisach-Mayergoyz (P-M) model, which was modified in a way that significantly improves its numerical performances.

\section{Computationally EfFicient Formulation OF THE PREISACH-MAYERGOYZ MODEL}

The 3-D vector extension of the Preisach model designed by Mayergoyz [2] has the reputation of having good physical accuracy but poor numerical performances [3]. The reason for this is that the P-M model works by combining the contributions of many Preisach operators oriented in different directions. For each of these Preisach operators, the Everett function must be evaluated at least once. This is usually achieved by performing a bicubic interpolation to ensure sufficient smoothness for the FE problem to converge [4]. The important number of operations that result from this procedure makes the computation time of the standard P-M model prohibitively large, especially for commercial FE software.

For this reason, we propose a new formulation of the P-M model, that is, specifically tailored for FE applications. We call this new formulation the computationally efficient
P-M (CEPM) model. Since the focus of this paper is on the incorporation of this model in an FE framework, we only present a brief overview of the model itself. A more detailed description of this new CEPM model is the subject of another paper yet to be published.

\section{A. Preisach Operator}

The scalar Preisach model can be described as an operator $\hat{\mathcal{P}}$ that takes an Everett function $E(\alpha, \beta)$, which can be identified by measuring a set of first-order reversal curves, and a magnetic field value $H$ as input, and outputs a magnetization value such that

$$
M(t)=\hat{\mathcal{P}}(E, H(t))
$$

The Preisach operator also records the history of the input field as an internal state that can be represented by a staircase boundary in the $\alpha-\beta$ domain, also known as the Preisach domain.

\section{B. Formulation of the CEPM Model}

The CEPM reuses Mayergoyz' idea to combine the contribution of many Preisach operators oriented in different directions to obtain a vector extension of the Preisach model. Each of these directions is defined in spherical coordinates by the unit vector

$$
\mathbf{e}_{\theta_{i}, \varphi_{i}}=\left[\sin \theta_{i} \cos \varphi_{i}, \sin \theta_{i} \sin \varphi_{i}, \cos \theta_{i}\right]^{\top} .
$$

The number and orientation of these unit vectors are specified by the Lebedev quadrature of the chosen degree $N_{L}$ to integrate the contributions of each Preisach operator. This quadrature is analogous to the Gauss-Legendre quadrature, but for integrals over a unit sphere surface. In fact, because of a symmetry in the P-M model, we must only integrate over half of a sphere. Therefore, only half the directions of a usual Lebedev quadrature must be considered.

The main difference with the standard P-M model is that instead of projecting the magnetic field vector $\mathbf{H}$ onto $\mathbf{e}_{\theta_{i}, \varphi_{i}}$ and then evaluating the Preisach operator with a nonlinear 
Everett function $N_{L}$ times, in the case of the CEPM, we first compute a nonlinear single-valued function $\mathbf{G}(\mathbf{H})$ and then project it onto every directions. This $\mathbf{G}(\mathbf{H})$ function is conveniently chosen so that, when it is used in combination with a very simple and quick to compute Everett function, it leads to the desired output. A reversible part noted $\mathbf{F}(\mathbf{H})$ is also added to the model. In mathematical terms, this translates to

$$
\mathbf{M}(\mathbf{H})=\mathbf{F}(\mathbf{H})+\sum_{i=1}^{N_{L}} w_{i} \frac{\mathbf{e}_{\theta_{i}, \varphi_{i}}}{B_{r}} \hat{\mathcal{P}}\left(E_{1}, \mathbf{e}_{\theta_{i}, \varphi_{i}} \cdot \mathbf{G}(\mathbf{H})\right)
$$

where $w_{i}$ is the weight associated with $i$ th direction in the Lebedev quadrature, $B_{r}$ is the magnetization at remanence, and $E_{1}$ is the bilinear Everett function defined as

$$
E_{1}(\alpha, \beta)= \begin{cases}0, & \alpha \beta \geq 0 \\ -\alpha \beta, & \alpha \beta<0 .\end{cases}
$$

In the isotropic case, we impose that the vector functions $\mathbf{F}$ and $\mathbf{G}$ be collinear with $\mathbf{H}$ and that their magnitude depends only on the magnitude of $\mathbf{H}$. Therefore, the isotropic CEPM model only requires the computation of two nonlinear scalar functions: $F(H)$ and $G(H)$.

Because this new formulation greatly reduces the number of evaluations of nonlinear functions compared to the standard $\mathrm{P}-\mathrm{M}$ model formulation, it turns out to be much more computationally efficient (hence the name). In order to quantify this speed-up, we implemented both versions of the model in Fortran and compared them outside the FE framework. Our test showed that, for a degree of the Lebedev quadrature $N_{L}=25$, the CEPM model is 132 times faster than the standard implementation of the P-M model. What is even more remarkable is that this speed-up is not achieved at the expense of numerical precision. On the contrary, by using the simple bilinear Everett function, the CEPM model reduces the integration error of the Lebedev quadrature by two orders of magnitude.

\section{Identification of the Parameters of the CEPM Model}

Another nice feature of the CEPM model is that its parameters can be identified easily using only the measurement of the major hysteresis loop. Assuming that the ascending branch $B_{a}(H)$ and the descending branch $B_{d}(H)$ of the major hysteresis loop are symmetric, such that

$$
B_{d}(H)=-B_{a}(-H)
$$

we can construct the functions $F(H)$ and $G(H)$ for values of $H$ greater than zero as

$$
F(H)=B_{d}(H)-B_{r}-\mu_{0} H
$$

and

$$
G(H)=B_{r}-\frac{B_{d}(H)+B_{d}(-H)}{2} .
$$

By construction, this method of identification allows the CEPM model to reproduce exactly the major hysteresis loop. Interestingly, it also models minor hysteresis loops with good accuracy. Indeed, we can see in Fig. 1 that the computed minor loops are close to the measured ones even though we

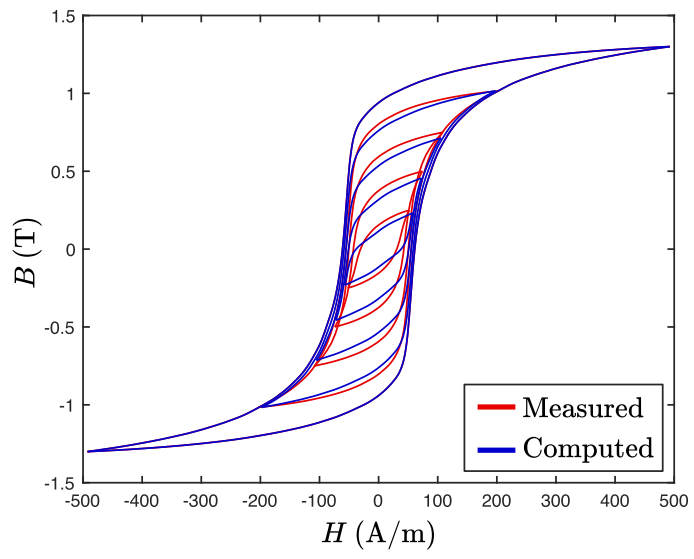

Fig. 1. Comparison between the measured concentric hysteresis loops and the computed ones using the CEPM model for the FeSi steel used in the TEAM problem 32 [5].

TABLE I

CEPM Model Parameters For the TEAM Problem 32

\begin{tabular}{cccc}
\hline$i$ & $a_{i}$ & $b_{i}$ & $c_{i}$ \\
& $(\mathrm{~T})$ & $(\mathrm{A} / \mathrm{m})$ & $(\mathrm{A} / \mathrm{m})$ \\
\hline 1 & 0.5043 & 11.08 & 59.37 \\
2 & 0.4162 & 130.19 & 114.39 \\
\hline
\end{tabular}

only used information about the major hysteresis loop for the identification process.

So far, we have expressed the functions $F(H)$ and $G(H)$ as a function of the descending branch of the major hysteresis loop $B_{d}(H)$ without specifying how it can be evaluated for arbitrary values of $H$. In order to be able to do just that, we propose to express the major hysteresis loop as

$$
B_{d}(H)=\mu_{0} H+\sum_{i=1}^{N} a_{i} \arctan \left(\frac{H+c_{i}}{b_{i}}\right) .
$$

Using this analytical expression, $3 N$ parameters are needed to describe the entire model. These parameters are found by fitting (8) on the measurement data of $B_{d}(H)$. We performed this procedure with the data of the TEAM problem 32 with a value of $N=2$ and obtained the parameters listed in Table I.

\section{IMPLEMENTATION OF THE CEPM MODEL IN AN FE SOFTWARE}

The CEPM model was implemented in the commercial FE software Altair Flux [10]. As it is presented in Section II, the CEPM model gives the material's magnetic property in the $\mathbf{B}(\mathbf{H})$ form that is compatible with the $\mathbf{T}-\phi$ formulation of Maxwell's equations that we use to solve 3-D magnetotransient FE problems. To be compatible with the A- $V$ formulation, the material law would need to be inverted.

Regarding the FE framework, the CEPM model is used at each Gaussian integration point of the magnetic region during the assembly. For each of these points, we first compute the local magnetization using (3) to construct the right-hand side vector and then compute the gradient of the magnetization using an analytical expression to construct the Jacobian matrix. Consequently, the relevant history for the CEPM model must be stored at the integration points. 


\section{IMPROVING THE NONLINEAR CONVERGENCE OF Finite Element Problems With Hysteresis}

The CEPM model possesses many characteristics that make it a good candidate for a vector hysteresis model in 3-D FE analysis: excellent physical accuracy, good numerical performances, simple identification method using limited experimental data, and description of the model using a small number of parameters. However, like other vector hysteresis models, it suffers from convergence issues when implemented in an FE framework. This is, in part, due to the discontinuity in the derivative of the B-H curve at reversal points [9].

In this section, we propose a transient nonlinear scheme designed to improve the robustness of the convergence and accelerate the solving time of FE problems with hysteresis. Some methods used in this scheme are already well known, but the novelty lies in the strategies employed to combine those methods together. Note that the application of this scheme is not limited to FE problems with hysteresis, it can also be applied to accelerate other transient nonlinear problems with convergence issues.

\section{A. Adaptive Time-Stepping Method}

We use a prediction-correction scheme [6] to automatically refine the time step size near reversal points. For each time step, the algorithm first calculates a prediction of the solution using a second-degree polynomial extrapolation. The real solution is then found by solving the nonlinear FE problem. The comparison between the prediction and the real solution allows to evaluate an error criterion that is used to refine the time step.

\section{B. Prediction of the Solution}

In standard transient nonlinear schemes, the nonlinear iterations are often initialized using the solution from the previous time step. This strategy usually makes sense, because the time step size is always chosen to be small compared to the characteristic time associated with the physical processes involved in the problem. Therefore, the solution does not change much from one time step to the next, so using the solution from the previous time step seems to be a reasonable approximation to initialize the nonlinear iterations.

In the case of problems with hysteresis, however, this choice is very problematic. Indeed, using the solution from the previous time step for initialization causes the nonlinear iterations to start exactly at the point of discontinuity in the derivative of the B-H curve (see Fig. 2). In order to fix this problem, we initialize the nonlinear iterations using the prediction of the solution already computed for the adaptive time stepping scheme. Moreover, because the prediction is obtained through a second-degree polynomial extrapolation, it is possible to anticipate a change in the derivative of the B-H curve at the reversal point. This strategy, though simple and computationally cheap, was found to greatly improve the convergence of FE problems with hysteresis.

\section{Newton-Raphson Method}

Although the Newton-Raphson (N-R) is often considered ill-suited for nonlinear problems with discontinuous

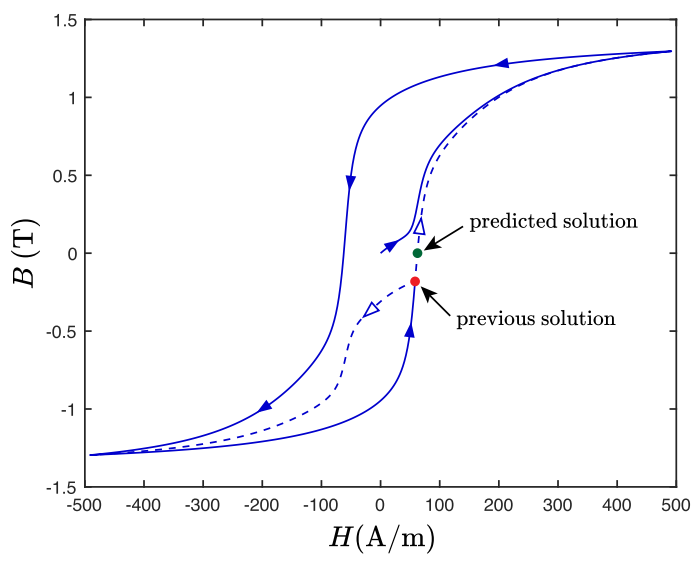

Fig. 2. Full blue curve represents the path that has already been computed, while the blue dashed line represents the two possible branches on which the next solution lies. Using the solution from the previous time step (red dot) initializes the nonlinear iterations at the point of discontinuity.

derivatives, it has already been applied to some hysteresis problems with success [7]. In our own numerical experimentations, it has been noticed that, for time steps that do not produce important variations in the direction of the magnetic field, the convergence using the N-R is indeed very quick.

For this reason, we choose an optimistic approach in our scheme and start the nonlinear iterative process with the $\mathrm{N}-\mathrm{R}$ method.

\section{Adaptive Relaxation Method}

The N-R method is fast, but it can sometimes converge to a local minimum or simply diverge. To add robustness to our nonlinear scheme, we also use an adaptive relaxation factor $\alpha$ computed using a line search method [8]. In our implementation of the method, a series of values of $\alpha$ is tried until we find one for which the norm of the residual is smaller than that of the previous nonlinear iteration. By ensuring that the residual decreases at every nonlinear iteration, the $\mathrm{N}-\mathrm{R}$ method is much more likely to converge.

\section{E. Local-Coefficient Fixed-Point Method}

Even with the adaptive relaxation method, the N-R method is not guaranteed to converge or it might do so only for very small values of $\alpha$ and thus very slow. For this reason, when the line search method fails to find a suitable relaxation factor (greater than a certain value $\alpha_{\min }$ ), we switch to a fixed-point method for the remaining nonlinear iterations of the current time step.

The specific fixed-point scheme we use is called the localcoefficient method (LCM). It was originally proposed by Dlala and Arkkio [9] to solve FE problems with magnetic hysteresis. With this method, the differential permeability tensor $\partial \mathbf{B} / \partial \mathbf{H}$ is replaced by the local fixed-point permeability which corresponds to the average between the largest and smallest terms of the main diagonal of the differential permeability tensor

$$
\mu_{F P}=\frac{1}{2}\left(\left.\frac{\partial \mathbf{B}}{\partial \mathbf{H}}\right|_{\min }+\left.\frac{\partial \mathbf{B}}{\partial \mathbf{H}}\right|_{\max }\right) \text {. }
$$

Although the convergence is slower with the LCM fixedpoint method than with the N-R method, it has proven to be very robust in our experience. 


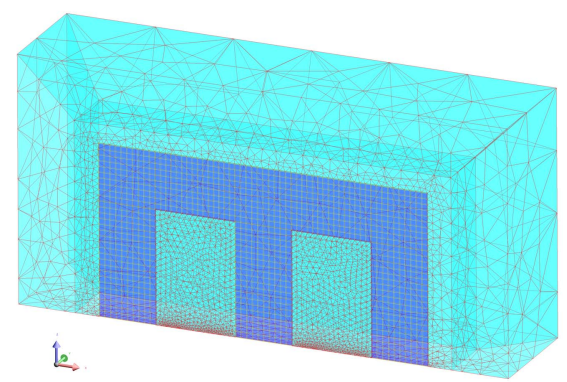

Fig. 3. Meshed geometry for the 3-D FE simulation of the TEAM problem 32 .

TABLE II

Convergence Results For the TEAM Problem 32 WiTH AND Without HYSTERESIS

\begin{tabular}{ccc}
\hline & w/ hysteresis & w/o hysteresis \\
\hline simulation time for 1 cycle & $2 \mathrm{~h} 41 \mathrm{~m}$ & $1 \mathrm{~h} 10 \mathrm{~m}$ \\
no. of time steps / cycle & 125 & 121 \\
average no. of iterations / time step & 10 & 5 \\
\hline
\end{tabular}

\section{Results For THE TEAM PROBLEM 32 IN 3-D}

For this paper, we simulated the TEAM problem 32 which is a benchmark specifically designed to test vector hysteresis models [5]. The set-up consists of a three-limb transformer with a ferromagnetic core made from a stack of five $0.48 \mathrm{~mm}$ thick non-grain oriented steel laminations. We simulated the test case no. 3 in which the two outer limbs are excited with voltage driven coils supplied by sinusoidal sources $(14.5 \mathrm{~V}$ peak) at $10 \mathrm{~Hz}$. The two signals are out of phase by $90^{\circ}$ which produces a rotating magnetic field at the T-joint of the transformer.

For the FE problem, we represented one-fourth of the full 3-D geometry and meshed it with 17030 elements (see Fig. 3). We used a magnetic scalar potential $\phi$-formulation since all regions are considered non-conducting, which leads to 33330 degrees of freedom. The material parameters we used for the CEPM model are the ones given in Table I.

We performed the simulation twice on a standard laptop computer. The first time with the CEPM model and the second time with an anhysteretic B-H curve described by (8) with the coefficients $a_{i}$ and $b_{i}$ taken from Table I, but with all the coefficients $c_{i}=0$. A comparison of the simulation time and convergence characteristics is presented in Table II. The results show that our transient nonlinear scheme enables the problem with hysteresis to be solved in almost the same number of time steps than that of the problem without hysteresis. However, because the hysteresis problem sometimes requires the use of the LCM fixed-point method, the average number of nonlinear iterations per time step is doubled as compared to the anhysteretic case. This results in an increase of the overall simulation time by a factor of 2.3 , which we consider to be very acceptable considering the significant physical complexity that is added to the problem by taking hysteresis effects into account.

The simulation results for the B-loci at the T-joint using the CEPM model are compared with the experimental data
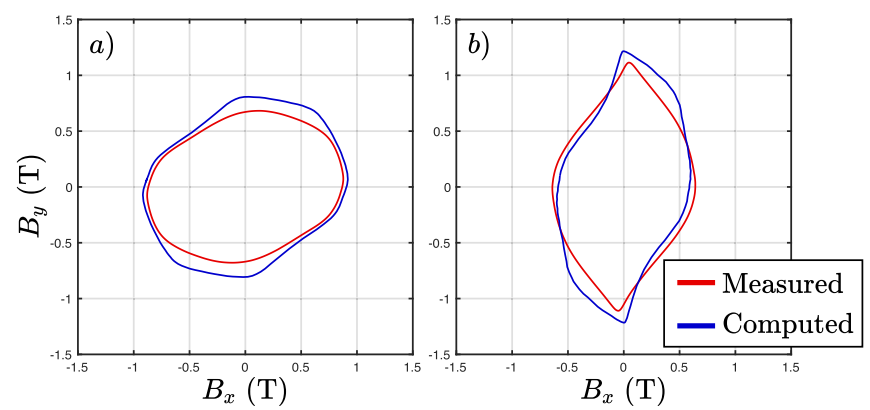

Fig. 4. Computed and measured B-loci at (a) pick-up coils $\mathrm{C} 1$ and $\mathrm{C} 2$ and (b) pick-up coils $\mathrm{C} 3$ and $\mathrm{C} 4$.

provided by Bottauscio et al. [5] in Fig. 4. We can see that the correspondence between the measured and computed curve is relatively good considering the level of difficulty of this problem. It must also be noted that the CEPM model was entirely identified using uniaxial measurements. It is, therefore, not so surprising to observe a small discrepancy between the simulation and the experiment for the case of rotational fields.

\section{CONCLUSION}

We presented a new computationally efficient formulation of the P-M model that was implemented in 3-D FE analysis. An improved transient nonlinear scheme was also developed to deal with the discontinuity in the derivative of the hysteresis curve. These new techniques allowed us to solve the TEAM problem 32 with hysteresis in a little more than twice the time it takes to solve the same problem without hysteresis.

\section{REFERENCES}

[1] K. Hoffmann, J. P. A. Bastos, J. V. Leite, and N. Sadowski, "A vector Jiles-Atherton model for improving the FEM convergence," IEEE Trans. Magn., vol. 53, no. 6, Jun. 2017, Art. no. 7300304.

[2] I. D. Mayergoyz, Mathematical Models of Hysteresis and their Applications, 1st ed. New York, NY, USA: Elsevier, 2003.

[3] L. Dupre and J. Melkebeek, "Electromagnetic hysteresis modelling: From material science to finite element analysis of devices," Int. Compumag Soc. Newslett., vol. 10, no. 3, pp. 4-15, 2003.

[4] E. Dlala, "Efficient algorithms for the inclusion of the Preisach hysteresis model in nonlinear finite-element methods," IEEE Trans. Magn., vol. 47 , no. 2, pp. 395-408, Feb. 2011.

[5] O. Bottauscio, M. Chiampi, C. Ragusa, L. Rege, and M. Repetto, "Description of TEAM problem 32: A test-case for validation of magnetic field analysis with vector hysteresis," Istituto Elettrotecnico Nazionale Galileo Ferraris, Turin, Italy, Tech. Rep., 2004. [Online]. Available: http://www.compumag.org/jsite/images/stories/TEAM/ problem32.pdf

[6] P. Massé, B. Morel, and T. Breville, "A finite element prediction correction scheme for magneto-thermal coupled problem during Curie transition," IEEE Trans. Magn., vol. 21, no. 5, pp. 1871-1873, Sep. 1985.

[7] J. Gyselinck, M. De Wulf, L. Vandevelde, and J. Melkebeek, "Incorporation of vector hysteresis and eddy current losses in 2D FE magnetodynamics," in Proc. ELECTRIMACS, vol. 99, no. 9, 1999, pp. $37-44$.

[8] K. Fujiwara, T. Nakata, N. Okamoto, and K. Muramatsu, "Method for determining relaxation factor for modified Newton-Raphson method," IEEE Trans. Magn., vol. 29, no. 2, pp. 1962-1965, Mar. 1993.

[9] E. Dlala and A. Arkkio, "Analysis of the convergence of the fixed-point method used for solving nonlinear rotational magnetic field problems," IEEE Trans. Magn., vol. 44, no. 4, pp. 473-478, Apr. 2008.

[10] Altair Flux. Access: 2019. [Online]. Available: https://altairhyperworks.com/product/flux 